\title{
Topiramate-induced ocular side effects in Egyptian patients, idiosyncratic versus dose-dependent effect, a prospective study
}

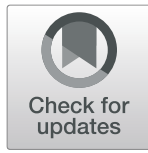

\author{
Mohamed N. Thabit ${ }^{{ }^{*}}$ (D) and Mahmoud Mohamed Farouk ${ }^{2}$
}

\begin{abstract}
Background: To test the nature of the ocular side effects induced by topiramate (TPM) whether dose dependent or idiosyncratic, and to test various predictors that might influence the occurrence of those side effects.

Methods: Twenty patients treated with TPM were included in this study. Patients underwent ophthalmic assessment before and after 4 weeks of treatment by stable doses of oral TPM. We examined non-cycloplegic refraction (RF) in diopters, best corrected visual acuity (BCVA), intraocular pressure (IOP) in $\mathrm{mmHg}$, and anterior chamber depth (ACD) in mm.

Results: There were no statistically significant differences between baseline and follow-up assessments in all tested ophthalmological parameters including errors of RF, ACD, IOP, and the BCVA. One case suffered from painful drop of vision in both eyes with elevated IOP and decreased ACD, and evident myopic shift 1 week after treatment with small dose of TPM. There was no significant effect of age, TPM dose, disease, and gender on all tested variables.

Conclusion: TPM can induce idiosyncratic, but not dose dependent, ocular side effects, namely myopic shifts and angle closure glaucoma. Those side effects were not disease, age, or gender dependent. However, ethnicity might play a role in induction of those side effects.
\end{abstract}

Keywords: Topiramate, Eye, Myopia, Glaucoma, Side effects

\section{Introduction}

Later in the previous century, in 1996, topiramate (TPM) was approved by the Food and Drug Administration (FDA) for treatment of epilepsy. TPM is a sulfaderivative monosaccharide that has powerful antiepileptic activity through various mechanisms, including blockage of voltage gated sodium channels, activation of potassium current inducing hyperpolarization, potentiation of post synaptic gamma aminobutyric acid receptors, inhibition of $\alpha$-amino-3-hydroxy-5-methyl-4isoxazolepropionic acid (AMPA)/kainite receptors, and finally inhibition of carbonic anhydrase enzyme [1-3]. TPM is characterized by rapid absorption and crossing

\footnotetext{
* Correspondence: Mohamed_hamdon@med.sohag.edu.eg 1 Department of Neurology, Sohag University Hospital, Sohag University, Madinat, Nasser, Sohag, Egypt

Full list of author information is available at the end of the article
}

of the blood brain barrier and is renally excreted with an elimination half-life of around $21 \mathrm{~h}[4,5]$. In 2004, the FDA approved the use of TPM for prevention of migraine [6]. Moreover, many other off-label indications of TPM have been published in various literature including bipolar disorder, post-traumatic stress disorder, obesity, eating disorders, idiopathic increased intracranial tension, essential tremors, and many others [7-11]. As discussed above, the spectrum of uses of TPM is widening and also its side effects' range is increasing. The most common adverse effects of TPM include sleepiness, fatigue, lack of concentration/cognitive problems, vertigo, agitation, nephrolithiasis, and metabolic acidosis [2, 12]. In particular, the eye side effects are of utmost importance, and neurologists, psychiatrists, and ophthalmologists must be fully aware of it. 
Many ocular adverse effects of TPM have been described including acute angle closure glaucoma, ocular pain and headache, red eye, mydriasis, acute onset myopia, uveitis, and choroidal effusion [3]. The most common ocular adverse effect of TPM is a ciliochoroidal effusion syndrome which can cause a spectrum of clinical manifestations ranging from TPM-induced myopic shift (TiMS) up to bilateral TPM-induced angle closure glaucoma (TiACG). Acute transient blurring of vision that resolves after drug cessation is the whole mark of TiMS. However, TiACG may be resistant to treatment whether topical of systemic and may eventually lead to permanent visual loss [13].

Most of the literature that has been published before to study the ocular adverse effects of TPM was case reports [13]. Only a few prospective studies have been published before. Leung et al. did not describe any significant changes in the depth of anterior chamber in a Chinese group of patients after 1-month use of a fixed dose $(50 \mathrm{mg} /$ day) of TPM [14]. Another two studies done for Turkish patients: one reported both acute (within 1 week) increase in the thickness of the ciliary body and decrease in the angle of the anterior chamber using a fixed dose of TPM $(50 \mathrm{mg} /$ day), and the other one found a significant increase in the intraocular pressure (IOP) and decrease in the anterior chamber depth (ADC) after 1 and 2 months of use of a fixed dose of TPM $(50 \mathrm{mg} /$ day) in patients with migraine $[15,16]$. Last study included an Iranian group of patients with migraine. This study reported only significant myopic shift without significant changes in ACD or IOP after 30 and 90 days of use of a $50 \mathrm{mg} /$ day of TPM [17]. Those prospective studies reported inconsistent results among different ethnic groups. Moreover, most of the studies used fixed dose schedule for most patients and most patients were suffering from migraine.

\section{Methods}

\section{Aim of the work}

The aim of our study was to test the ocular parameters that might be related to TiMS, and TiACG in a wider dose range of TPM, different diseases, and different ethnic group, Egyptian patients. This aimed to answer the missing questions in the previous studies, namely, are the ocular changes induced by TPM dose dependent?, different among various ethnic groups?, related to age or gender?, and different diseases? or not.

\section{Subjects}

Twenty-eight patients suffer from diseases in whom TPM is indicated, namely, patients with epilepsy and migraine were recruited from the Neurology clinic in our university hospital. Only twenty patients (38 eyes) were included in this study and the rest of the patients dropped out during follow-up period. Six patients discontinued the drug in the titration phase due to other non-ocular side effects and two patients did not follow up with our clinic. Patients were sent to the Ophthalmology clinic for baseline assessment of the tested ocular parameters. Patients were tested again after 1 month of stable usage of the therapeutic dose of TPM excluding the titration phase which was around 2 weeks. Followup assessment was done by the same examiner (MF) and same devices. The treatment period of TPM of ranged from one and half to 2 months elapsed between baseline and follow-up assessments. During this period, weekly follow-up for drug titration and monitoring the response to or the side effects of TPM was done during the first 2 weeks up to 1 month. At least 1 month of treatment with TPM in a stable therapeutic dose elapsed between baseline and follow-up assessments. The age, gender, stable therapeutic doses of TPM, and the disease from which the patients were suffering were registered for each patient. Patients with history of previous intraocular surgery or glaucoma were excluded from the study. All the study participants gave an informed consent for participation in the study and the study protocol was approved by local health committee of our university

\section{Ophthalmological assessment}

All patients underwent ophthalmic assessment at baseline (before starting the treatment) and after 1 month of receiving a stable dose of oral TPM. The assessment included history of ocular diseases, medications, and surgery. In each visit, the patients were examined for noncycloplegic refraction (RF) by Auto Ref/Keratometer (HRK-7000A, Huvitz, Korea), best corrected visual acuity (BCVA), IOP measured by I-care ic100 tonometer (ICare Finland Oy, Helsinki, Finland), anterior segment examination by slight lamb, fundus examination, and ACD measurement by A-scan ultrasonography (Digital A/B scan 5500; Sonomed Inc., Lake Success, NY, USA).

The main outcome measures were the spherical equivalent RF in diopters (D), BCVA in Snellen decimal notation, IOP in $\mathrm{mmHg}$, and ACD in $\mathrm{mm}$.

\section{Statistical analysis}

The mean \pm SD and the percentage were used for presentation of numerical and categorical data, respectively. Testing the normality of the numerical data was done using the Kolmogorov-Smirnov test. The statistical package SPSS for Windows (Version 16) was used for statistical analysis. Paired sample $T$ test and univariate repeated measures ANOVA were used to determine the significant differences between groups for the numerical data. Chi-squared test and Wilcoxon-signed rank tests were used for categorical data and non-normally 
distributed numerical data. A $P$ value of $<0.05$ was considered statistically significant.

\section{Results}

The demographic variables of the study participants are presented in Table 1 . The mean \pm SD of various variables of ophthalmological assessments and its statistical significance are also presented on Table 2. As regards the RF, 21 eyes were myopics, 15 eyes were hypermetropics, and 4 eyes were immetropics. There were no statistically significant differences between baseline and follow-up assessments in all ophthalmological assessment including errors of RF, ACD, IOP, and the BCVA (Table 2).

One case (female patient, 23 years old) suffered from painful drop of vision in both eyes 1 week after starting treatment with TPM. On examination, the BCVA dropped from 0.7 to 0.1 in both eyes, the RF changed from $-1.50 \mathrm{D}$ to $-6.00 \mathrm{D}$ in the right eye and from $1.75 \mathrm{D}$ to $-5.50 \mathrm{D}$ in the left eye, the IOP was elevated from 16 to $27 \mathrm{mmHg}$ in both eyes, and the ACD decreased from 3.6 to $3.1 \mathrm{~mm}$ in both eyes. This case was managed by stoppage of the oral TPM and starting antiglaucoma medications in the form of topical beta blockers $(0.5 \%$ timolol $)$ for 2 weeks. The parameters were normalized within 1 week and the topical beta blocker was not needed to maintain normal IOP after 2 weeks.

Univariate repeated measures ANOVA taking the baseline and follow-up assessments as the within-subject factors, the gender (males vs females) and disease (epilepsy vs migraine) as between-subjects variables, and finally the age and the stable TPM dose as covariates were done. There was no significant main effect of age, stable TPM dose, disease, and gender on all tested variables (Table 3).

\section{Discussion}

The aim of this study was to test the ocular side effects, namely those related to the ciliochoroidal effusion syndrome, of TPM in Egyptian patients, and moreover to test the effect of the various doses, various indications, age, and gender of the patients on the chance to develop those side effects. We found that TPM did not induce any significant asymptomatic, dose, indication, age, or gender dependent changes in the RF, IOP, ACD, and BCVA in those study participants. However in our study, we reported one case with acute onset of painful drop of vision in both eyes shortly after the start of treatment with TPM. These acute changes rapidly resolved after discontinuation of TPM and use of the relevant medications. In this case, both TiMS and TiACG associated with decreased ACD were evident. The co-existence of these complications can be explained by the syndrome of ciliochoroidal effusion discussed below. The ocular side effects occurred in this case were idiosyncratic. We described this effect as "idiosyncratic" as it occurred with a very small dose, $25 \mathrm{mg} /$ day in a patient who was still in the dose titration phase, and in just one case among the study participants. Moreover, there are some evidences of severe intoxication with TPM without occurrence of TiACG [12].

The findings of our study were in agreement with many previous case reports [18-25] and one of the previous published prospective studies [14]. On the contrary, other studies described changes that were not found in our study [15-17]. Those studies found significant ocular side effects induced by TPM including changes in the size of the ciliary body, ACD, RF, and IOP. The differences between the findings of our study and those studies might be in part explained by racial differences or genetic factors between the studies' participants. The difference in the ethnic groups might also explain the difference between the previous studies itself. Leung et al. did not find any significant changes in a Chinese group of patients, like our study; however, the other two studies done for Turkish patients and the one done for Iranian patients found some significant changes in various ophthalmological parameters [14-17].

TPM is a sulfa derivative antiepileptic drug [13]. Other sulfa derivative drugs as sulfamethizole, chlortalidone, hydrochlorothiazide, sulfapyridine, trimethoprim, and acetazolamide can also induce MS and ACG [3]. One controversial hypothesis states that TPM induces swelling of the lenses. This swelling might be responsible alone or with other factors about the occurrence of TiMS and TiACG [26, 27]. Another hypothesis postulated that a form of swelling occurs in the ciliary body, hence the term ciliochoroidal effusion syndrome; this swelling potentially cause anterior rotation of the ciliary

Table 1 Demographic data for patients participated in the study

\begin{tabular}{ll}
\hline Presumed predictor & Patients receiving TPM \\
\hline Age (years) & $26.2 \pm 10.3$ \\
Sex & 8 males $(40 \%) ; 12$ females (60 \%) \\
Disease & $10(50 \%)$ patients suffered epilepsy and the other 10 (50\%) suffered migraine \\
Stable TPM dose & $7(35 \%)$ used $50 \mathrm{mg} /$ day, $8(40 \%)$ used $100 \mathrm{mg} /$ day, and 5 (25\%) used $200 \mathrm{mg} /$ day
\end{tabular}


Table 2 Data for ophthalmological assessment for all patients

\begin{tabular}{|c|c|c|c|c|c|}
\hline & & $\begin{array}{l}\text { Baseline } \\
\text { Mean } \pm \text { SD }\end{array}$ & Follow-up Mean \pm SD & $T$ value & $P$ value \\
\hline \multirow[t]{2}{*}{$\mathrm{RF}(\mathrm{D})$} & Myopics & $-1.1 \pm 0.6$ & $-1.5 \pm 1.6$ & 1.52 & 0.138 \\
\hline & Hypermetropics & $1.1 \pm 1.7$ & $1.1 \pm 1.7$ & & \\
\hline \multicolumn{2}{|c|}{$\mathrm{ACD}(\mathrm{mm})$} & $3.6 \pm 0.2$ & $3.5 \pm 0.3$ & 1.27 & 0.212 \\
\hline \multicolumn{2}{|c|}{ IOP (mmHg) } & $14.1 \pm 3$ & $14.8 \pm 4.2$ & -1.62 & 0.114 \\
\hline \multicolumn{2}{|c|}{ BCVA (Snellen) } & $0.8 \pm 0.2$ & $0.8 \pm 0.2$ & 1.43 & 0.16 \\
\hline
\end{tabular}

$R F$ refraction in diopters, $A C D$ anterior chamber depth, $I O P$ intraocular pressure, $B C V A$ best corrected visual acuity

processes, narrowing of the ciliary sulcus, and forward displacement of the iris diaphragm, which all together lead to the occurrence of TiACG. Furthermore, the swelling of the ciliary body leads to the relaxation of the zonules of the lens leading to its thickening and the occurrence of TiMS [28].

\section{Conclusions}

In conclusion, we found that TPM, although can induce idiosyncratic ocular side effects, did not induce any dose, disease, age, or gender asymptomatic changes in the RF, ACD, IOP, or BCVA in most patients. Accordingly, when prescribing this drug for treatment of various diseases, the patients must be well informed about the occurrence of acute unexpected ocular side effects even with the use of small doses. And if this happens, the drug must be discontinued immediately and an ophthalmologist's advice must be taken.

Table 3 Main effect of age, drug dose, disease, and gender on different variables of ophthalmological assessment

\begin{tabular}{llll}
\hline Variable & Predictors & $\boldsymbol{F}$ value & $\boldsymbol{P}$ value \\
\hline RF & Dose & .149 & .702 \\
& Age & 1.091 & .305 \\
& Indication & 2.421 & .131 \\
ACD & Gender & .911 & .348 \\
& Dose & .295 & .591 \\
& Age & 1.444 & .240 \\
IOP & Indication & 1.203 & .282 \\
& Gender & .483 & .493 \\
& Dose & .176 & .678 \\
& Age & 2.899 & .100 \\
BCVA & Indication & 5.252 & .030 \\
& Gender & .224 & .639 \\
& Dose & 1.273 & .269 \\
& Age & 1.897 & .212 \\
& Indication & 2.689 & .112 \\
\hline & Gender & .365 & .551 \\
\hline
\end{tabular}

$R F$ refraction in diopters, $A C D$ anterior chamber depth, $I O P$ intraocular pressure, $B C V A$ best corrected visual acuity

\section{Limitations of the study}

One limitation of our study is the small sample size which may lower the statistical power. Further largescale multicenter studies including patients from different ethnic groups are still needed.

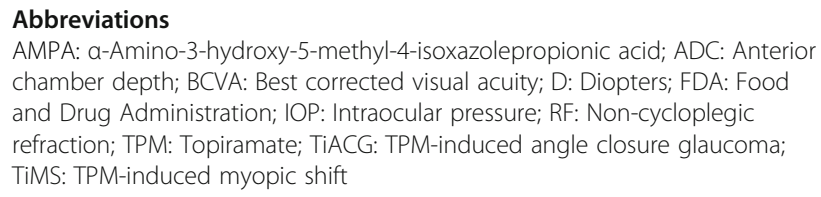

AMPA: a-Amino-3-hydroxy-5-methyl-4-isoxazolepropionic acid; ADC: Anterior chamber depth; BCVA: Best corrected visual acuity; D: Diopters; FDA: Food and Drug Administration; IOP: Intraocular pressure; RF: Non-cycloplegic refraction; TPM: Topiramate; TiACG: TPM-induced angle closure glaucoma; TiMS: TPM-induced myopic shift

\section{Acknowledgements \\ We would like to thank all who helped us in this work especially our colleagues including Dr Ahmad Ezat, Dr Mostafa Abdelmomen, and Dr Ahmad El-shanhory for their help and support in this research.}

\section{Prior presentation}

This work has not been previously presented.

\section{Authors' contributions}

MF analyzed and interpreted all ophthalmology-related data. MT selected the patients and disease classification and demographic data of the patients and controls. All authors participated in writing the manuscript, and all authors read and approved the final manuscript.

\section{Funding}

This research received no specific grant from any funding agency in public, commercial, or non-profit sectors.

\section{Availability of data and materials}

The datasets used and/or analyzed during the current study are available from the corresponding author on reasonable request.

\section{Ethics approval and consent to participate}

All study participants signed an informed written consent, and the study protocol was approved by the local ethics committee of Sohag University Hospital. The reference number of this committee is not available.

\section{Consent for publication}

This manuscript does not contain any individual person's data in any form, including individual details, images, or videos.

\section{Competing interests}

None of the authors has any financial conflict of interest relating to this manuscript.

\section{Author details}

'Department of Neurology, Sohag University Hospital, Sohag University, Madinat, Nasser, Sohag, Egypt. 'Department of Ophthalmology, Sohag University Hospital, Sohag University, Sohag, Egypt. 
Received: 31 August 2020 Accepted: 17 December 2020

Published online: 07 January 2021

\section{References}

1. Angehagen M, Ronnback L, Hansson E, Ben-Menachem E. Topiramate reduces $A M P A$-induced $\mathrm{Ca}(2+)$ transients and inhibits GluR1 subunit phosphorylation in astrocytes from primary cultures. J Neurochem. 2005; 94(4):1124-30.

2. Mirza NS, Alfirevic A, Jorgensen A, Marson AG, Pirmohamed M. Metabolic acidosis with topiramate and zonisamide: an assessment of its severity and predictors. Pharmacogenet Genomics. 2011;21(5):297-302.

3. Panday VA, Rhee DJ. Review of sulfonamide-induced acute myopia and acute bilateral angle-closure glaucoma. Compr Ophthalmol Update. 2007; 8(5):271-6.

4. Christensen J, Hojskov CS, Dam M, Poulsen JH. Plasma concentration of topiramate correlates with cerebrospinal fluid concentration. Ther Drug Monit. 2001;23(5):529-35.

5. Perucca E. A pharmacological and clinical review on topiramate, a new antiepileptic drug. Pharmacol Res. 1997:35(4):241-56.

6. Ramadan NM, Buchanan TM. New and future migraine therapy. Pharmaco Ther. 2006;112(1):199-212.

7. Mula M, Cavanna AE, Monaco F. Psychopharmacology of topiramate: from epilepsy to bipolar disorder. Neuropsychiatr Dis Treat. 2006;2(4):475-88.

8. Berlin HA. Antiepileptic drugs for the treatment of post-traumatic stress disorder. Curr Psychiatry Rep. 2007;9(4):291-300.

9. McElroy SL, Guerdjikova Al, Martens B, Keck PE Jr, Pope HG, Hudson Jl. Role of antiepileptic drugs in the management of eating disorders. CNS Drugs. 2009;23(2):139-56.

10. Celebisoy N, Gokcay F, Sirin H, Akyurekli O. Treatment of idiopathic intracranial hypertension: topiramate vs acetazolamide, an open-label study. Acta Neurol Scand. 2007;116(5):322-7.

11. Leblhuber F, Steiner K. Topiramate treatment of essential tremor in a patient with cognitive deficits. Case Rep Neurol. 2010;2(1):1-4.

12. Wisniewski M, Lukasik-Glebocka M, Anand JS. Acute topiramate overdose-clinical manifestations. Clin Toxicol (Phila). 2009:47(4):317-20.

13. Abtahi MA, Abtahi SH, Fazel F, Roomizadeh P, Etemadifar M, Jenab K, et al. Topiramate and the vision: a systematic review. Clin Ophthalmol. 2012;6: 117-31.

14. Leung DY, Leung H, Baig N, Kwan P, Kwong YY, Wong KS, et al. Topiramate and asymptomatic ocular angle narrowing: a prospective pilot study. Eye (Lond). 2009;23(11):2079-81.

15. Gok M, Ozdemir O. Effect of topiramate on choroidal thickness and anterior chamber parameters in the treatment of patients with migraine. Cutan Ocul Toxicol. 2017;36(4):381-6.

16. Karalezli A, Koktekir BE, Celik G. Topiramate-induced changes in anterior chamber angle and choroidal thickness. Eye Contact Lens. 2016:42(2):120-3.

17. Hesami O, Hosseini SS, Kazemi N, Hosseini-Zijoud SM, Moghaddam NB, Assarzadegan F, et al. Evaluation of ocular side effects in the patients on topiramate therapy for control of migrainous headache. J Clin Diagn Res. 2016;10(3):NC01-4.

18. Agarwal A. Ciliochoroidal effusion in topiramate-induced bilateral acute angle closure glaucoma. Indian J Ophthalmol. 2019;67(9):1466-7.

19. Czyz CN, Clark CM, Justice JD, Pokabla MJ, Weber PA. Delayed topiramateinduced bilateral angle-closure glaucoma. J Glaucoma. 2014;23(8):577-8

20. Joshi AK, Pathak AH, Patwardhan SD, Kulkarni AN. A rare case of topiramate induced secondary acute angle closure glaucoma. J Clin Diagn Res. 2017; 11(6):ND01-ND3.

21. Lam L, Wang N, Ormonde S, Merriman M. Topiramate-induced secondary angle closure: salient points in management. Clin Exp Optom. 2018;101(5): 704-6.

22. Lan YW, Hsieh JW. Bilateral acute angle closure glaucoma and myopic shift by topiramate-induced ciliochoroidal effusion: case report and literature review. Int Ophthalmol. 2018;38(6):2639-48.

23. Mahendradas P, Parab S, Sasikumar R, Kawali A, Shetty BK. Topiramateinduced acute angle closure with severe panuveitis: a challenging case report. Indian J Ophthalmol. 2018;66(9):1342-4.

24. Osaba M, Reviglio VE. Case report: the role of OCT in examination of a patient with topiramate-induced acute angle closure, acute myopia and macular striae. Oxf Med Case Reports. 2018;2018(7):omy030.

25. Sierra-Rodriguez MA, Rodriguez-Vicente L, Chavarri-Garcia JJ, Del Rio-Mayor $J$. Acute narrow-angle glaucoma induced by topiramate with acute myopia and macular striae: A case report. Arch Soc Esp Oftalmol. 2019;94(3): 130-3.

26. Sen HA, O'Halloran HS, Lee WB. Case reports and small case series: topiramate-induced acute myopia and retinal striae. Arch Ophthalmol. 2001; 119(5):775-7.

27. Craig JE, Ong TJ, Louis DL, Wells JM. Mechanism of topiramate-induced acute-onset myopia and angle closure glaucoma. Am J Ophthalmol. 2004; 137(1):193-5.

28. Ikeda N, Ikeda T, Nagata M, Mimura O. Ciliochoroidal effusion syndrome induced by sulfa derivatives. Arch Ophthalmol. 2002;120(12):1775.

\section{Publisher's Note}

Springer Nature remains neutral with regard to jurisdictional claims in published maps and institutional affiliations.

\section{Submit your manuscript to a SpringerOpen ${ }^{\circ}$ journal and benefit from:}

- Convenient online submission

- Rigorous peer review

- Open access: articles freely available online

- High visibility within the field

- Retaining the copyright to your article

Submit your next manuscript at $\boldsymbol{\nabla}$ springeropen.com 